\title{
Alexander Calder's Half-Circle, Quarter-Circle, and Sphere (1932): a complex history of repainting unraveled
}

\author{
Federica Pozzi ${ }^{1 *}$, Julie Arslanoglu ${ }^{1}$ and Eleonora Nagy ${ }^{2}$
}

\begin{abstract}
The Whitney Museum of American Art, New York, owns one of the largest motorized works made by the renowned American artist Alexander Calder, titled Half-Circle, Quarter-Circle, and Sphere. Created in 1932, and acquired by the Whitney in 1969, this seminal work was featured in an iconic exhibition held in 2017 and entitled Calder: Hypermobility. Prior to that, the object underwent a series of treatments in order to repair its main kinetic elements that had become compromised during its lifetime. While the work's mechanism retained its creator's ingenious engineering solutions, the motor, urethane belts, plug, and electrical wires turned out to be neither original, nor authentic to the period. The appearance of the piece had also been altered, as most surfaces displayed multiple layers of overpainting and, thus, did not deliver the proper gloss, hue, and texture. These observations prompted a first, comprehensive scientific study to investigate the stratigraphy of Calder's painted surfaces on Half-Circle, Quarter-Circle, and Sphere, with the final goal to comprehend and restore its original appearance through careful removal of the overpaint. Non-invasive X-ray fluorescence (XRF) analysis was carried out to gain initial insight into the paints' composition. After that, extensive microscopic sampling was performed to assess the possible presence of original layers below the repainting throughout the object's surface. Cross sections were examined with optical microscopy and analyzed with Fourier-transform infrared (FTIR) and Raman spectroscopies, as well as scanning electron microscopy coupled with energy-dispersive X-ray spectroscopy (SEM/EDS), in order to identify pigments, colorants, and extenders located in the various paint layers. Scrapings were also investigated with pyrolysis-gas chromatography/mass spectrometry (Py-GC/ MS) for a detailed characterization of the binding media. Scientific analysis revealed, in selected white and red areas, up to eleven layers of overpaint composed of a wide array of modern materials, including pigments (titanium white in the form of tetragonal rutile and a variety of synthetic organic red pigments) and binders (alkyd or late formulations of enamels based on ortho-phthalic acid/phthalic anhydride, glycerol and pentaerythritol, polyvinyl acetate with various plasticizers, and acrylics). On the other hand, the identification of materials that were available in the early 1930s, such as zinc white, calcite, and gypsum, as well as traditional drying oil binders, supported the hypothesis that a layer of original paint may still be present in certain areas. In addition to shedding new light on the stratigraphy of Calder's painted surfaces, this study informed the optimization of a treatment plan tailored for the safe removal of the overpaint to uncover the original layer, wherever present.
\end{abstract}

Keywords: Alexander Calder, Motorized sculpture, Modern art, Stratigraphy, Overpainting

\footnotetext{
*Correspondence: federica.pozzi@metmuseum.org

${ }^{1}$ Department of Scientific Research, The Metropolitan Museum of Art, 1000 Fifth Avenue, New York, NY 10028, USA

Full list of author information is available at the end of the article
}

\section{Introduction}

Alexander Calder is one of the best-known and most beloved American artists. Born in 1898 into a family of artists, he was trained as an engineer at Stevens Institute of Technology in New Jersey but, in 1923, while on the west coast of Canada, he decided to become an artist

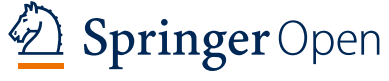

(c) The Author(s) 2020. This article is licensed under a Creative Commons Attribution 4.0 International License, which permits use, sharing adaptation, distribution and reproduction in any medium or format, as long as you give appropriate credit to the original author(s) and the source, provide a link to the Creative Commons licence, and indicate if changes were made. The images or other third party material in this article are included in the article's Creative Commons licence, unless indicated otherwise in a credit line to the material. If material is not included in the article's Creative Commons licence and your intended use is not permitted by statutory regulation or exceeds the permitted use, you will need to obtain permission directly from the copyright holder. To view a copy of this licence, visit http://creativeco mmons.org/licenses/by/4.0/. The Creative Commons Public Domain Dedication waiver (http://creativecommons.org/publicdomain/ zero/1.0/) applies to the data made available in this article, unless otherwise stated in a credit line to the data. 
himself. By the fall of 1926, he left New York for Paris, then the global hub of modernity as well as tradition, fashion, pleasure and, most of all, art. Compared to the United States' increasing isolationism, prohibition, puritanism, and racial division, the French capital offered enticing artistic liberties and freedoms. It was there that Calder became acquainted with many famous artists, including Mondrian, Picasso, Miro, Duchamp, Kertész, and Matisse, and made connections with a number of galleries that provided the perfect environment for the budding artist.

While experimenting with drawing, painting, woodcarving, and wire in the late 1920 s in Paris, Calder became interested in motion. In the fall of 1929, he visited Mondrian's studio and later recalled: "This one visit gave me a shock that started things. I suggested to Mondrian that perhaps it would be fun to make these rectangles oscillate" [1]. Revolutionary thoughts followed: "Why must art be static? You look at an abstraction, sculpted or painted, an exciting arrangement of planes, spheres, nuclei, entirely without meaning. It would be perfect, but it is always still. The next step in sculpture is motion" [2]. As a result, Calder's signature sculptural medium would become not the traditional stone or clay, but motion: kinetics in space.

Greatly influenced by the dramatic astronomical discoveries around 1930, including the identification of Pluto as the ninth planet in the solar system, Calder started building motorized works in 1931. His first creation, now destroyed, was entitled Motorized Mobile That Duchamp Liked. In October 1931, Marcel Duchamp visited Calder's studio in Paris and saw one of his motordriven works, with three elements. As Calder recalled: "I had just painted it, and he was so anxious to see it move that he pushed it, and he got all full of paint [3]. I asked him what sort of a name I could give these things and he, at once, produced «mobile»" [1]. In addition to indicating something that moves, this word in French also means «motive».

Currently, fewer than 40 motorized mobiles of similar scale are known, all made by Calder in a span of 10 years. $\mathrm{He}$ created entire compositions with several elements rotating at different relative speed. A single cycle of these motorized sculptures would take from a few minutes to up to $45 \mathrm{~min}$ to complete. One of the largest and earliest whimsical works, Half-Circle, Quarter-Circle, and Sphere (referred to as Half-Circle from here onwards), belongs to the collection of the Whitney Museum of American Art, New York. Created in 1932, Calder titled this mobile simply by naming the shapes it comprises. The sphere, an implied form created by the intersection of two red painted disks, swings as an upside-down pendulum, while the black-painted heavy-gage metal rod that forms the half circle and quarter circle element rotates around its own axis. The white base-an essential part of the object-is the visual anchor of the work, as well as the physical support that houses the motor. The white color of the base allows the moving elements of the sculpture to be the focus of the spectator's attention rather than the base.

Besides their physical appearance, motion is a vital part of Calder's mobiles. The artist admitted that his motorized contraptions were not made for eternal perfection. In a 1932 interview for the New York World-Telegram, Calder reasoned his tinkering: "I had my choice between perfecting a motor for one or two things, or going on to new creations. I preferred to go on creating". He added: "To an engineer, good enough means perfect" [2]. Hence, the mechanics inside Half-Circle's base are strikingly imprecise. Calder's joyful personality and his deep vein of hilarity, embodied in his figurative works, is also present in his machineries. Indeed, the motion of these gadgets often includes one or more surprise moments, such as an unexpected change of speed or an apparent collision of the moving elements. In Half-Circle, in particular, the black rod first appears to kick the side of the sphere and, then, the two elements almost crash into one another.

Acquired by the Whitney Museum in 1969, over three decades after its creation, Half-Circle has travelled and operated extensively. It functioned intermittently until 2010, when it was sent to the Art Gallery of Ontario, Canada, the last venue of the Alexander Calder: The Paris Years, 1926-1933 retrospective exhibition, after which it was no longer operational. A 2016 restoration treatment involving the mechanism revealed that almost all mechanical glitches were caused by loose bits of wood, belts, wire, and nails that were not inherent to the motor itself. The current motor, dated to 1980 and recycled from an old photocopy machine, was likely selected because it fit the cradle of the missing original motor, about which no information could be recovered. The speed of motion of the red sphere and black rod21 and $14 \mathrm{~s}$, respectively-locates Half-Circle within the speed range of similar mobiles. Accuracy of the relative velocity of the spiral to the sphere is supported by one of Calder's drawings of Half-Circle's twin piece Double Arc and Sphere, also dated to 1932: on this drawing, the artist indicated the sphere's swing to be slower than the spin of the spiral, and that the pulleys gear down the speed of the motor, which corresponds exactly to what is observed in Half-Circle. Moreover, in Calder's opinion, the speed of a mobile's motion was not a rigid rule. For instance, he recalled changing his mobiles' speed at the $1932 \mathrm{Gal}$ lery Villon exhibition: “[Mondrian] said they weren't fast enough, and when I stepped on things, he said they weren't fast enough, so I said I'd make one especially fast 
to please him, and then he said that that would not be fast enough-because the whole thing ought to be still" [4]. Thus, despite the non-original motor, Half-Circle likely retained the right type and speed range of its motion; only the exact original speed of the individual elements remains, so far, unknown.

The painted surfaces of Calder's Half-Circle posed another significant challenge. Verbal accounts and conservators' experience through practice narrate that the artist typically painted his works with commercial household products, applying the paint straight from the can, directly onto the metal or wood, with no surface preparation or primer coat $[5,6]$. He allegedly favored very matte paints and applied thin, single layers by means of a brush. According to such non-written sources, occasionally, Calder himself applied the paint or, in other cases, recommended a caretaker to restore a mobile. Orrin Riley, founder of the conservation department at the Solomon R. Guggenheim Museum and chief restorer there for more than two decades, recalled Calder giving him a bucket of red paint to repaint Red Lily Pads, the iconic mobile hanging from the museum's spiraling rotunda [7]. These accounts attest to a long history of excessive overpainting and, in some cases, complete repainting of Calder's works that, over the years, has prompted much debate in the art conservation world. For decades, when a paint became worn or chipped, lacunae were typically left and the entire surface would be repainted, reportedly often with the same or similar color or paint brand, as also observed on Half-Circle.

As in the case of several other Calder objects, during its over 85 years of life, the appearance of this mobile, too, has been considerably altered. Most of its surfaces are currently overpainted and, as a result, do not deliver the proper gloss, hue, and texture as originally intended by the artist. The existing thick, gleaming white paint, for instance, is far from Calder's matte, single-layer brush application of white colors, while the heavily overpainted red of the sphere does not even remotely resemble the thinly brushed original surface. In general, the thick, rough accumulation of layers observed in Half-Circle and other works appears extremely different from the fine, thin brush marks found on Calder's original painted surfaces, which causes the current appearance of this object to be overall inconsistent with its intended aesthetic. The above-mentioned history of overpainting and repainting of Calder's works, along with the lack of published analytical data on his materials and techniques, provided an impetus to document, recover, and retain any extant original paints on this noteworthy early mobile. With these observations in mind, an in-depth technical study was undertaken to gain insight into the stratigraphy of HalfCircle and address any outstanding issues in preparation for an exhibition, entitled Calder: Hypermobility, held at the Whitney Museum from June 9th to October 23rd, 2017 (Fig. 1). The present article reports for the first time a comprehensive, multi-technique scientific investigation of one of Calder's indoor motorized sculptures, Half-Circle, Quarter-Circle, and Sphere, with the threefold goal of gathering detailed information on the number and composition of paint layers in selected areas of the object; of determining whether an original layer might be present underneath the white, red, and black overpaint; and of informing the development and optimization of a treatment plan tailored for the safe removal of the overpaint to uncover the original layer, wherever present.

\section{Experimental}

The extensive campaign of scientific analysis performed on Calder's Half-Circle included both in situ noninvasive investigations with portable instruments and removal of microscopic samples followed by analysis with benchtop equipment in the Department of Scientific Research (DSR) of The Metropolitan Museum of Art (The Met). Special attention was devoted to inspecting the paints' composition in relation to the possible presence of an original paint layer underneath

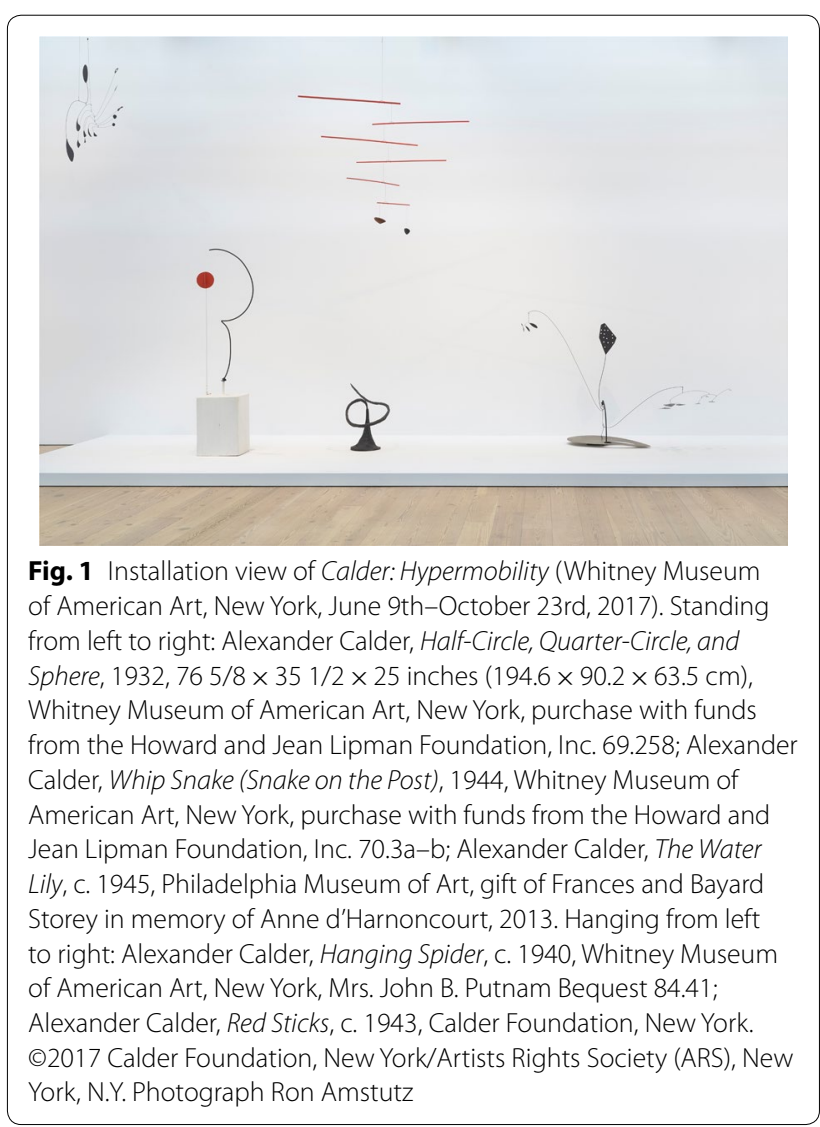


the repainting. Initially, non-invasive analysis was carried out at the Whitney Museum storage facility using a handheld X-ray fluorescence (XRF) spectrometer, in order to gain preliminary information on the elements contained in the white, red, and black paints in a selection of representative locations. Removal of fifteen scrapings and samples for cross sections led to examination with optical microscopy and analysis with a variety of instrumental techniques, including transmission and attenuated total reflection (ATR)-Fourier-transform infrared (FTIR) spectroscopy, Raman spectroscopy, as well as scanning electron microscopy coupled with energy-dispersive X-ray spectroscopy (SEM/EDS), with the main goal to identify the pigments, colorants, and extenders located in the various paint layers. Loose samples were also investigated with pyrolysis-gas chromatography/mass spectrometry (Py-GC/MS) for a detailed characterization of the binding media present within the stratigraphy. Experimental conditions for the analytical techniques used are reported in the following section.

$X R F$ Analysis was performed using a handheld Bruker Tracer III- $\mathrm{V}^{\mathrm{TM}}$ energy dispersive XRF analyzer, with Peltier-cooled advanced high-resolution silver-free Si-PIN detector with a $0.2-\mu \mathrm{m}$ beryllium $(\mathrm{Be})$ window and average resolution of approximately $142 \mathrm{eV}$ for the full width at half maximum of the manganese $(\mathrm{Mn}) \mathrm{K} \alpha$ line. The system is equipped with changeable filters, and a rhodium (Rh) transmission target with maximum voltage of $45 \mathrm{kV}$ and tunable beam current of 2-25 $\mu \mathrm{A}$. The size of the spot analyzed is approximately $3 \times 4 \mathrm{~mm}$. Analysis was performed using $40 \mathrm{kV}, 12.5 \mu \mathrm{A}, 120$-s acquisition time, and a titanium ( $\mathrm{Ti}$ )-aluminum (Al) filter, by positioning the instrument at $\mathrm{a} \approx 1-\mathrm{mm}$ distance from the artwork's surface.

SEM/EDS Analysis was carried out with a FE-SEM Zeiss igma HD equipped with an Oxford Instrument $\mathrm{X}$-MaxN 80 silicon drift detector (SDD). Backscattered electron (BSE) imaging and EDS elemental analysis were performed in high vacuum at $20 \mathrm{kV}$, on 12-nm carboncoated samples.

Raman Analysis was conducted using a Bruker Senterra Raman spectrometer equipped with Olympus $50 \times$ and $100 \times$ long working distance microscope objectives and a charge-coupled device (CCD) detector. A continuous wave diode laser, emitting light at $785 \mathrm{~nm}$, was used as the excitation source, and two holographic gratings (1800 and 1200 rulings $/ \mathrm{mm}$ ) provided a spectral resolution of $3-5 \mathrm{~cm}^{-1}$. The output laser power was kept between 10 and $25 \mathrm{~mW}$, while the number of scans and integration time were adjusted to prevent damage from overheating and according to the Raman response of the samples examined. Spectra were interpreted by comparison with published literature and library databases available at The Met's DSR.

FTIR Analysis was performed with a Hyperion 3000 FTIR spectrometer equipped with a mercury cadmium telluride (MCT) detector. For measurements in transmission, each sample was crushed in a Spectra Tech diamond anvil cell and all paint layers contained in it were analyzed as a bulk through a $15 \times$ objective. For ATR measurements, each paint layer in the cross sections was analyzed individually by means of a $20 \times$ ATR objective featuring a germanium crystal. In both cases, spectra were collected in the $4000-600 \mathrm{~cm}^{-1}$ range at a resolution of $4 \mathrm{~cm}^{-1}$ as the sum of 128 or 256 scans, depending on the response of the various samples. Spectra were interpreted by comparison with published literature and library databases available at The Met's DSR.

$P y$-GC/MS Analysis was carried out on an Agilent $5973 \mathrm{~N}$ gas chromatograph equipped with a Frontier PY-2020iD Double-Shot vertical furnace pyrolyzer fitted with an AS-1020E Auto-Shot autosampler. The GC was coupled to a $5973 \mathrm{~N}$ single quadrupole mass selective detector (MSD). Samples of 30-50 $\mu \mathrm{g}$ were weighed out in deactivated pyrolysis sample cups (PY1EC80F Disposable Eco-Cup LF) on a Mettler Toledo UMX2 Ultra microbalance. Samples were then either pyrolyzed without derivatization or derivatized with tetramethyl ammonium hydroxide (TMAH) before pyrolysis. Derivatization took place in the same cups as follows: $3-4 \mu \mathrm{l}$ of $25 \% \mathrm{TMAH}$ in methanol (both from Fisher Scientific), depending on the sample size, were added directly to the sample in each cup with a $50-\mu \mathrm{L}$ syringe and, after $1 \mathrm{~min}$, loaded onto the autosampler. The interface to the $\mathrm{GC}$ was held at $320^{\circ} \mathrm{C}$ and purged with helium for $30 \mathrm{~s}$ before opening the valve to the GC column. The samples were then dropped into the furnace and pyrolyzed at $550{ }^{\circ} \mathrm{C}$ for $30 \mathrm{~s}$. The pyrolysis products were transferred directly to a DB-5MS capillary column $(30 \mathrm{~m} \times 0.25 \mathrm{~mm} \times 1 \mu \mathrm{m})$ with the helium carrier gas set to a constant linear velocity of $1.5 \mathrm{~mL} /$ min. Injection with a 30:1 split was used, in accordance with the sample size. The GC oven temperature program was: $40{ }^{\circ} \mathrm{C}$ for $1 \mathrm{~min} ; 10{ }^{\circ} \mathrm{C} / \mathrm{min}$ to $320^{\circ} \mathrm{C}$; isothermal for $1 \mathrm{~min}$. The Agilent 5973N MSD conditions were set as follows: transfer line at $320^{\circ} \mathrm{C}$, MS Quad $150{ }^{\circ} \mathrm{C}$, MS Source $230^{\circ} \mathrm{C}$, electron multiplier at approximately $1770 \mathrm{~V}$; scan range 33-550 amu. For samples run with TMAH, the detector was turned off until $3 \mathrm{~min}$ to avoid saturation by excess of derivatizing agent and solvent. Data analysis was performed on an Agilent MSD ChemStation D.02.00.275 software by comparison with the NIST 2005 spectral libraries. 


\section{Results and discussion White paints}

Careful inspection of Calder's Half-Circle revealed that different white paints are present on the object's surface. For instance, an uncharacteristically thick, high-gloss, multi-layer paint was applied on the entire exterior of the base with perpendicular brush strokes. Rounding all the sharp edges of the support and hiding the texture of the wood, this paint displays heavy drips and multiple times overpainted lacunae throughout the surface. On the other hand, thin, brush-applied white layers were visually identified as possible original paint for the base exterior, as was a different kind of paint used for the shelf and woodblock holding the pulley in the interior of the base. Slight shade variations in Calder's whites are not surprising, as the artist was known to often repurpose various bits of materials, which may have been already painted. Both the shelf and woodblock for the pulley do not seem to be integral part of the wooden case: they appear to have been machine cut and manufactured for other purposes, then chopped up to parts, and incorporated into the sculpture.

Two single-layered samples, S7 and S8, of what was visually recognized as likely original white paint were removed from the interior of the artwork's wooden base on the proper right and left sides, respectively, for scientific analysis. According to Raman and FTIR data, both samples contain mixtures of zinc white, barite and/or lithopone, calcite, and gypsum, which are accountable for the white color observed (Fig. 2). Analysis of the binding media with Py-GC/MS primarily found glycerol and a series of fatty acids, the evaluation of whose ratios represents a common method to differentiate among various oil media as well as describe modern adulterated oils and alkyds [8,9]. The most significant fatty acids found via the transesterification of triglycerides and esterification of free fatty acids are palmitic (P) and stearic (S) acids, as well as the diacids azelaic (A), suberic (Sub), and sebacic (Seb) acids. The ratio of $\mathrm{A} / \mathrm{P}$ reflects whether the oil analyzed might constitute a drying, semi-drying, or non-drying oil, while $\mathrm{P} / \mathrm{S}$ provides information on the exact identity of the drying oil. While traditional drying oils (e.g. linseed, walnut, poppy seed) have a fairly strict range of ratios, oils that have been adulterated with heavy metal soaps or other agents in order to achieve certain physical or mechanical properties can display widely ranging ratios. In samples $\mathrm{S} 7$ and $\mathrm{S} 8$, the fatty acid ratios $(\mathrm{P} / \mathrm{S}=1.3$ and $2.2, \mathrm{~A} / \mathrm{P}=0.86$ and 0.89 , respectively) are consistent with the presence of a traditional drying oil binder, possibly linseed oil (Fig. 3). These results clearly indicate, for samples S7 and S8, the use of a relatively limited array of pigments, extenders, and binding media that were all available in the early 1930s, when the artwork was created. Based on this observation, a hypothesis may be put forward that such a white layer could in fact represent an original paint applied by Calder to the piece's wooden base.
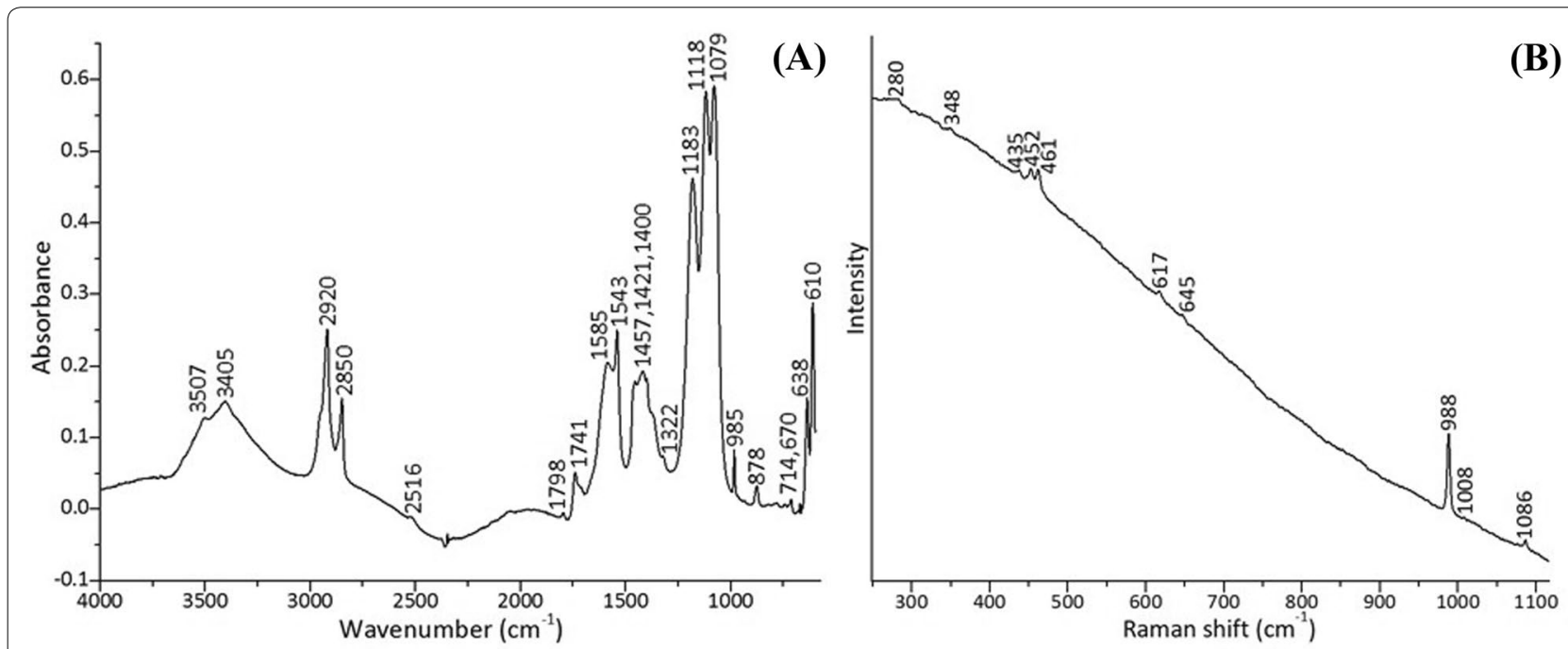

Fig. 2 FTIR and Raman spectra of sample S7 (likely original white). a FTIR spectrum of sample S7, i.e. single-layered white paint removed from the interior of the artwork's wooden base on the proper right. $\mathbf{b}$ Raman spectrum $\left(\lambda_{\text {exc }}=785 \mathrm{~nm}\right)$ of the same sample. Materials identified in the spectra include barite and/or lithopone (1183, 1118, 1079, 985, 638, $610 \mathrm{~cm}^{-1} \mathrm{FTIR} ; 348,452,461,617,645,988 \mathrm{~cm}^{-1}$ Raman), calcite $(2516,1798,1421,878$, $714 \mathrm{~cm}^{-1}$ FTIR; 280, $1086 \mathrm{~cm}^{-1}$ Raman), gypsum (3507, 3405, $670 \mathrm{~cm}^{-1}$ FTIR; $1008 \mathrm{~cm}^{-1}$ Raman), zinc white (435 $\mathrm{cm}^{-1}$ Raman), calcium oxalates (1322 $\left.\mathrm{cm}^{-1} \mathrm{FTIR}\right)$, zinc and other metal carboxylates $\left(2920,2850,1585,1543,1457,1400 \mathrm{~cm}^{-1} \mathrm{FTIR}\right)$, and an organic medium $\left(2920,2850,1741 \mathrm{~cm}^{-1}\right.$ FTIR) 


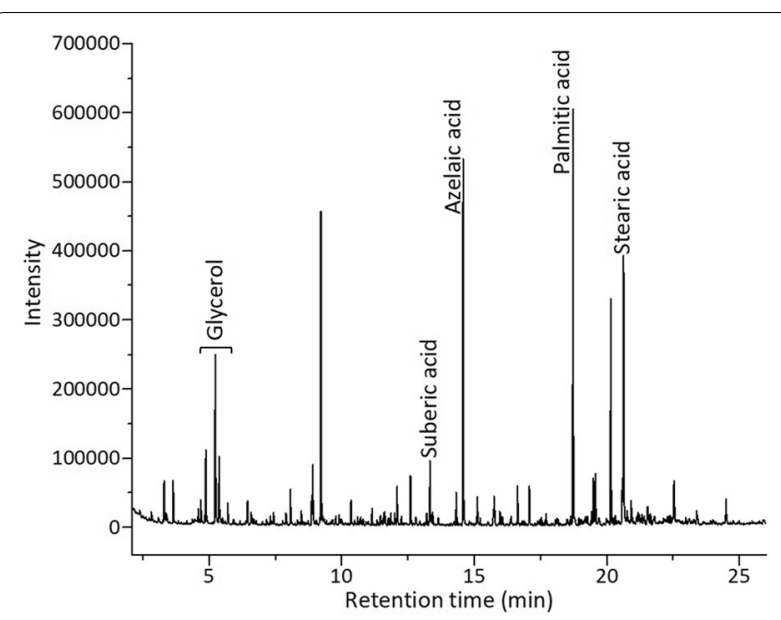

Fig. 3 Py-GC/MS chromatogram of sample S7 (likely original white). Py-GC/MS chromatogram obtained upon derivatization with TMAH of sample S7, i.e. single-layered white paint removed from the interior of the artwork's wooden base on the proper right. Compounds identified in the chromatogram and fatty acid ratios suggest the presence of a drying oil binder, possibly linseed oil (palmitic acid methyl ester $74,87,43,55,41,143,75,57,69,227,270 ;$ stearic acid methyl ester $74,87,43,55,75,143,41,57,69,298$; azelaic acid dimethyl ester 152, 55, 74, 83, 111, 59, 143, 185, 69, 41, 217)

On the other hand, two additional samples of singlelayered white paint, S9 and S10, taken from the internal shelf and woodblock in the artwork's mechanism, were found to be composed of lead white and calcite (S9), and of barite and/or lithopone, calcite, gypsum, and quartz (S10). With fatty acid ratios calculated as $\mathrm{P} / \mathrm{S}=1.4$ and $\mathrm{A} / \mathrm{P}=1.6$, results for white paint sample $\mathrm{S} 10$ show the presence of a drying oil modified by the addition of driers. All of the materials detected in samples S9 and S10 were also well known and widely used in the first few decades of the 20th century, which, again, may support authenticity of the paint layers examined.

In general, the use of different pigments in the white paints-zinc and lead whites, among others-may be explained by taking into consideration Calder's inclination towards a spontaneous use of materials and odd bits of wood at hand to create his works [1]. It is worth noting that, in three of the white paint samples mentioned above, the detection of trace amounts of abietic acid derivatives in the binding media pointed to the presence of a diterpenoid resin belonging to the Pinaceae family, which may be interpreted as either studio debris or as a deliberate addition to the oil to modify its properties. Additionally, zinc and other metal carboxylates, as well as calcium oxalates, were identified in many of the white paints examined. As often reported in the literature, the metal carboxylates might have been intentionally added to the oil binder to adjust properties such as pigment suspension, gelation, lubrication, and plasticizing [1012 ], and/or might originate from the chemical reaction between the oil and certain pigments in the paint layer. The oxalates, on the other hand, may result from the degradation of organic materials and their reaction with calcium-containing pigments and/or particulate dirt [13].

Examination of cross sections with optical microscopy and scientific analysis by means of SEM/EDS, Raman, and ATR-FTIR provided crucial information on the stratigraphy of Calder's painted surfaces in Half-Circle. In particular, cross sections S1, S4, and S5-all removed from white areas of the exterior of the base likely to have been repainted-display an overall similar, incredibly complex stratigraphy that comprises up to eleven distinct paint layers. Close inspection of the polarized light and ultraviolet (UV) light microphotographs, along with BSE images, revealed that these paint layers are characterized by an array of different colors (ranging from white and cream tones to various gray shades), UV-induced auto-fluorescence emissions, as well as pigment particle size and morphology, which are reflected by different elemental and chemical compositions (Fig. 4). Materials detected include a wide array of white pigments and additives, including titanium white, zinc white, barite and/or lithopone, calcite, gypsum, bassanite and/or anhydrite, dolomite, magnesite, silica, alumina, magnesia, as well as talc and various other silicates. In addition, a few iron(III) hydroxide inclusions as well as bone or ivory black particles were identified in most of the gray paint layers. In all three cross sections, titanium white is present in the form of tetragonal rutile, one of the three polymorphs of titanium dioxide along with tetragonal anatase and orthorhombic brookite. Interestingly, in cross sections S4 and S5, analysis with Raman spectroscopy detected a characteristic luminescence emission pattern that has been recently attributed to $\mathrm{Nd}^{3+}$ ions substituting into the orthorhombic alkaline earth sulfates of composite titanium dioxide pigments produced by co-precipitation with $\mathrm{BaSO}_{4}$ or $\mathrm{CaSO}_{4}$ [14]. In addition to delivering fundamental details related to pigment formulation and methods of manufacture, this observation bears interesting implications for dating. Indeed, although experimental rutile pigments were patented in 1931 in Czechoslovakia, Germany, and the United States, the first commercially viable methods for production were developed in 1937 and industrial manufacture began in 1938-39. Moreover, the anatase and calcium sulfate composite, introduced in the United States in 1925 , was phased out in the early 1940 s after rutile and calcium sulfate composites with increased hiding power and chalk resistance were introduced [15]. In cross sections $\mathrm{S} 4$ and S5, a rutile co-precipitated pigment was detected in most paint layers, including the bottom layers 

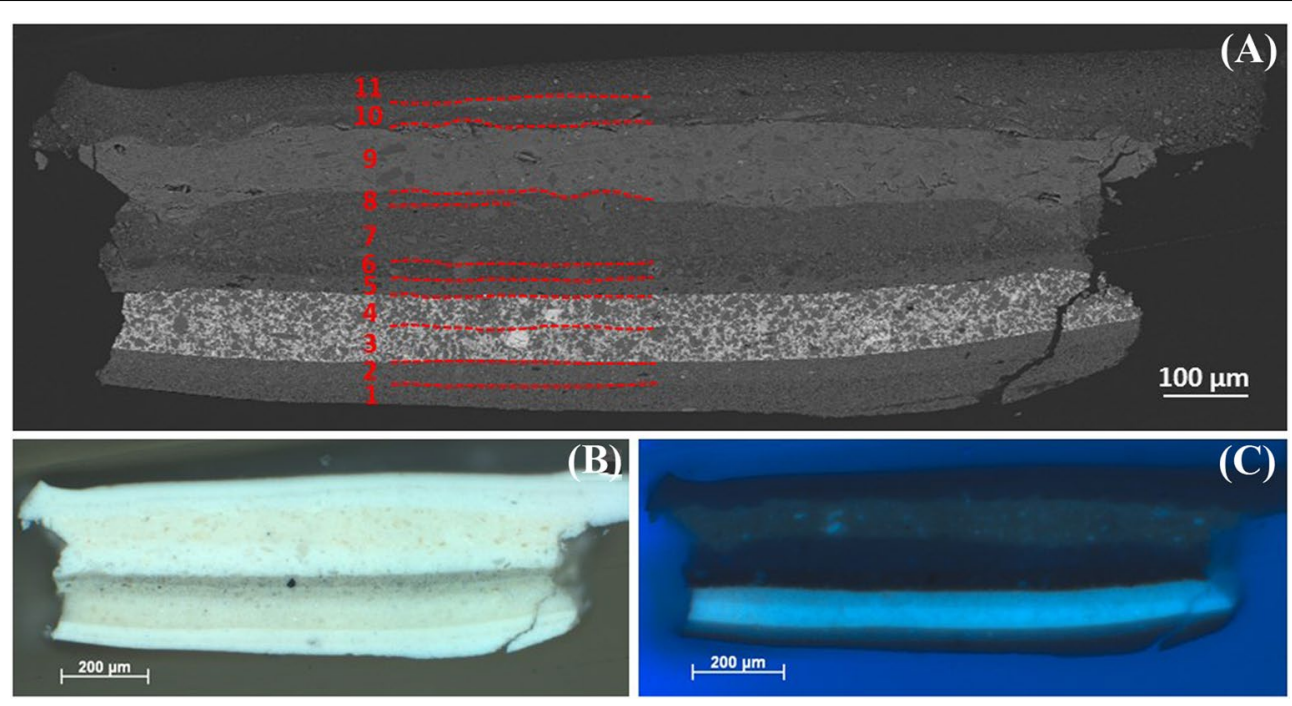

Fig. 4 BSE and optical microscopy images of cross section S4 (likely overpainting whites). a BSE image of cross section S4, i.e. multi-layered white paint removed from the artwork's exterior, on the proper right side, close to the base's rear edge at bottom, revealing eleven layers of overpaint. b Polarized and c UV light photographs of the same cross section, displaying different white shades as well as fluorescence emission colors and intensities for the various paint layers

(layers 1 and 2) (Fig. 5); on the other hand, the lowermost layer (layer 1) of cross section S1 was found to be similar in morphology and chemical composition to layers $3 / 4$ in S4 and layer 3 in S5, indicating that the stratigraphy in sample S1 is likely incomplete. Based on these observations, it can be hypothesized that all paint layers in cross sections S1, S4, and S5 were applied within later repainting campaigns, possibly to cover lacunae, which is in accordance with the initial visual assessment of the corresponding sampling sites.

Binding media analysis with Py-GC/MS provided additional insight into the possible dates of application of the paint layers found in these cross sections. Samples S2 and S3, corresponding to cross sections S1 and S4, respectively, contain a drying oil modified by a possible addition of a non-drying oil or metal palmitates $(\mathrm{P} / \mathrm{S}=1.3$ and $1.0, \mathrm{~A} / \mathrm{P}=0.45$ and 0.65 , respectively), alongside small amounts of a diterpenoid resin belonging to the Pinaceae family. In addition, the relatively high amounts of acetic acid and benzene liberated in the analysis of both samples are indicative of the presence of a polyvinyl acetate (PVAc) binder in one or more of the paint layers. Significant quantities of phthalates and phthalic anhydride were also identified in these two samples' chromatograms. The detection of the latter compounds could be either related to the oil and resin, thus indicating the use of an alkyd paint, i.e. late formulation enamels based on orthophthalic acid, or assigned to an early formulation of PVAc with phthalates used as external plasticizers [16]. As an additional alternative, it cannot be ruled out, based on
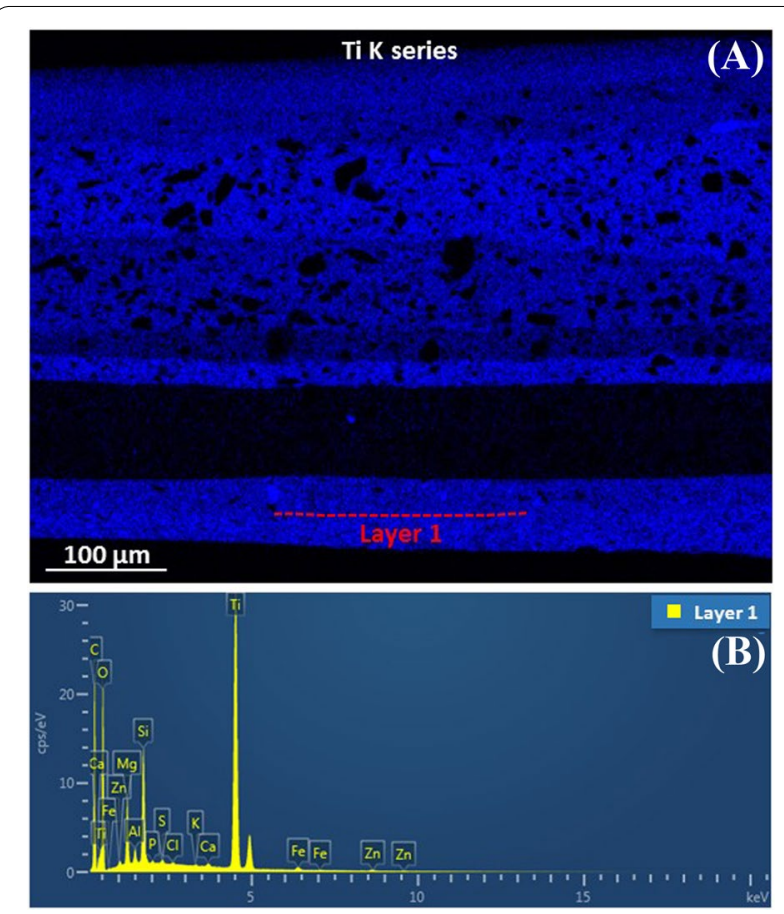

Fig. 5 X-ray elemental map of titanium and EDS spectrum of layer 1 in cross section S4 (likely overpainting whites). a X-ray elemental map of titanium collected from a portion of cross section S4, i.e. multi-layered white paint removed from the artwork's exterior, on the proper right side, close to the base's rear edge at bottom, highlighting the presence of this element-likely in the form of titanium white-in layer 1. b EDS spectrum of a portion of layer 1, confirming in this area the presence of relatively high amounts of titanium along with other elements 
the data collected, that the phthalates identified might bear a twofold attribution and be present in both components. Py-GC/MS analysis also found, in samples S2 and S3, relatively high amounts of styrene, which could point to the presence of styrene-modified alkyds or styrenated oils (Fig. 6) [16, 17]. On the other hand, the exact source of the trace levels of acrylic compounds detected in S3 remains unclear. Sample S6, a scraping of white paint corresponding to cross section S5, was found to contain a small oil component and Pinaceae resin, which may suggest the presence of an early enamel paint $[12,18]$. Due to the low intensity of the fatty acid peaks in the chromatograms, their ratios, in this case, cannot be used to draw conclusions on the type of oil present. As observed for samples S2 and S3, PVAc was also identified here, as well as phthalates and styrene, whose presence, as discussed above, may be associated with one or more of the binding media found in this sample. Furthermore, traces of acrylic compounds appear to be present in one or more of the paint layers. One final connection between S2, S3, and S6 is a tightly packed group of unique compounds detected between 9.1 and $10 \mathrm{~min}$ in the TMAH chromatograms-predominantly branched fatty acidswhich would appear to support the premise that these samples contain a particular paint formulation that is different from others analyzed on the sculpture.

While enamels were introduced in the late 19th century, all other binders mentioned above became commercially available in America at different times during the first half of the 20th century. The first oil-modified alkyd resins were produced in 1927, when DuPont began its research into decorative paints based on alkyds. However, it is not until shortly before the Second World War that alkyd-based paints were introduced into the American

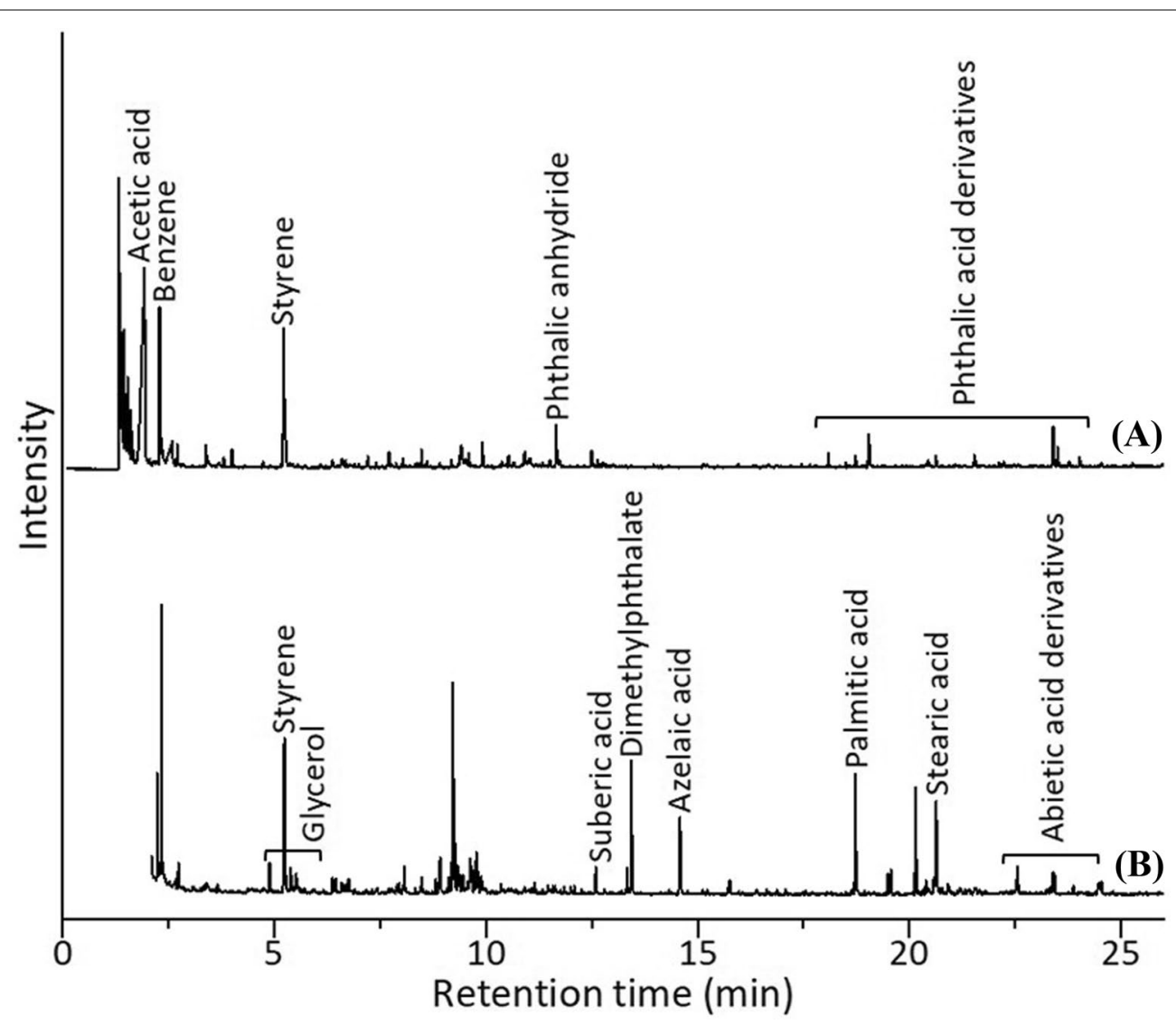

Fig. 6 Py-GC/MS chromatogram of sample S3 (likely overpainting whites). Py-GC/MS chromatograms obtained (a) without and (b) with derivatization with TMAH of sample S3, i.e. scraping of white paint removed from loss on the artwork's exterior, on the proper right side, close to the base's rear edge at bottom. Compounds identified in the chromatogram suggest the presence of an alkyd, PVAc, phthalates, and traces of acrylics (palmitic acid methyl ester 74, 87, 43, 55, 41, 143, 75, 57, 69, 227, 270; stearic acid methyl ester 74, 87, 43, 55, 75, 143, 41, 57, 69, 298; azelaic acid dimethyl ester 152,55,74, 83, 111,59, 143, 185, 69, 41, 217; styrene $\underline{104}, 103,78,51,77,50,105,52,102,63$; acetic acid 43, 45, 60, 42, 41; benzene $\underline{78}, 77,51,50,52,39,79,74,76,38$; phthalic anhydride 104, 76, 50, 148, 74, 75, 38, 77, 37, 105; phthalic acid, butyl 2-ethylhexyl 149, 41, 43, 150, 223, $57,55,205,76,334$; phthalic acid dimethyl ester $163,7,76,92,164,50,133,194,104,135 ;$ methyl methacrylate 41, 69, 39, 100, 99, 40, 59, 38, 85, 55; n-butyl methacrylate $69,87,41,56,39,55,57,70,142$ ) 
market, where they made a significant impact starting from the late 1950s. Generally, alkyd resins received only limited attention by manufacturers of artists' paints, with the notable exception of Winsor \& Newton's Griffin paints in 1970, and it is mainly in the form of house paint that they are found in works of art. In an attempt to overcome their susceptibility to hydrolysis, alkyds started to be modified with a variety of molecules, including styrene and acrylics, in the 1940s. Undocumented for use in decorative paints, styrenated alkyds were reportedly employed for low-cost fast-drying enamels, baking coats, and undercoats. Similarly, research on styrenated oils came to fruition in the late years of the same decade. First introduced in the 1930s, PVAc became available as a water-borne emulsion in the late 1940s and was initially available upon modification with external plasticizers, such as dibutyl phthalate or tricresyl phosphate, which, however, caused paint films to become weak and brittle. PVAc-based paints did not gain popularity in the United States until the mid-1950s, when the vinyl acetate monomer became financially competitive and resins were internally plasticized by copolymerizing vinyl acetate with a softer monomer. By that time, PVAc films were also being modified with alkyds in an attempt to improve their scrub resistance, freeze-thaw stability, and pigmentwetting properties. As with the alkyds, PVAc has had fairly limited use in the artists' paint market; the first artists' PVAc emulsion paint, reportedly developed by Burden Co. in 1945, never achieved success among artists due to issues with the effective distribution of pigments in the medium. Acrylics solutions and emulsions, on the other hand, were first introduced in the late 1940s and mid-1950s, respectively, and have been widely available in the form of artists' paints since their first introduction $[16,17,19,20]$. Based on this historical information and on the pigments' results previously discussed, and given that the presence of PVAc in the bottom layer (layer 1) of cross section S4 was unambiguously confirmed by ATRFTIR, the earliest date of application of the numerous paint layers observed in cross section S4 may be placed in the late 1940s. Application of the white paint layers found in the three cross sections examined within later repainting campaigns (post 1940) is also supported by the existing scholarship on Calder, as the use of both titanium white and PVAc-based media appears to be uncharacteristic for this artist [5-7].

\section{Red paints}

Wrinkled, thickly applied red paint with glossy appearance and multiple overpainting on large losses suggested that the sphere's red color is not authentic. Though Calder used different shades of bright orange-red in his works, the red color of Half-Circle appears somewhat deep, and certainly does not correspond to the thin brushstrokes seen on the artist's original reds. This paint showed a tendency to gum up and crumble, and does not reveal the simple construction of the intersected sheet metal circles and their mechanical joins as observed in original surfaces.

Cross sections S11 and S12, removed from the sphere's red flange and circle, display a nearly identical stratigraphy that includes seven paint layers consisting of an assortment of synthetic organic red pigments, a lead chromate-based pigment that is present as two distinctively orange layers located in the top portion of both cross sections, a cadmium sulfoselenide-based pigment in between them, as well as a variety of extenders, such as barite, gypsum, calcite, dolomite, silicates, silica, and alumina (Fig. 7). Four of the synthetic organic reds identified, i.e. PR1, PR3, PR4, and PR49, belong to the molecular class of $\beta$-naphthols, which are based on the coupling of a substituted aniline ring with $\beta$-naphthol and are among the oldest of synthetic organic pigments. While PR1 was the only coloring material detected in layer 2 of both cross sections, binary combinations of the other synthetic organic reds, sometimes along with inorganic pigments, appear to be predominant in all other paint layers, as follows: PR4 and PR49 in layers 1 and 3; a mixture of PR3 and PR4 with a lead chromate-based pigment in layers 5 and 7 (Fig. 8); and a cadmium sulfoselenidebased pigment, alongside traces of lead chromate, in layer 6 (Fig. 9). Interestingly, layer 4 was found to contain PR83 - the synthetic counterpart of natural dye alizarin (1,2-dihydroxyanthraquinone)-precipitated onto an alumina substrate (Figs. 8 and 9); accordingly, such layer in both S11 and S12 shows a translucent appearance that is typically associated with lake pigments. Among the $\beta$-naphthols, PR49, discovered in the late 19th century by Austrian chemist Paul Julius and also known as Lithol Red, was the first to be manufactured as a pigment lake and was widely available by the early 1920s; originally precipitated onto an inorganic carrier material such as barium sulfate, it was later used in its pure form [21]. In the present case, X-ray elemental maps collected by SEM/EDS display an abundance of calcium (Ca) throughout layers 1 and 3 of both samples S11 and S12, suggesting that the PR49 pigment used here might belong to the Ca type (PR49:2), the most commonly found along with the barium $(\mathrm{Ba})$ type (PR49:1) since the beginning of the manufacturing process (Fig. 9). Unfortunately, this hypothesis could not be confirmed by Raman spectroscopy, as spectra of the various salts $(\mathrm{Na}, \mathrm{Ca}$, and $\mathrm{Ba})$ of Lithol Red display nearly identical patterns, in which even band shifts in the region around $1200 \mathrm{~cm}^{-1}$ [22] fall within the calibration range and, thus, cannot be used to differentiate among the three lake pigments. Albeit 

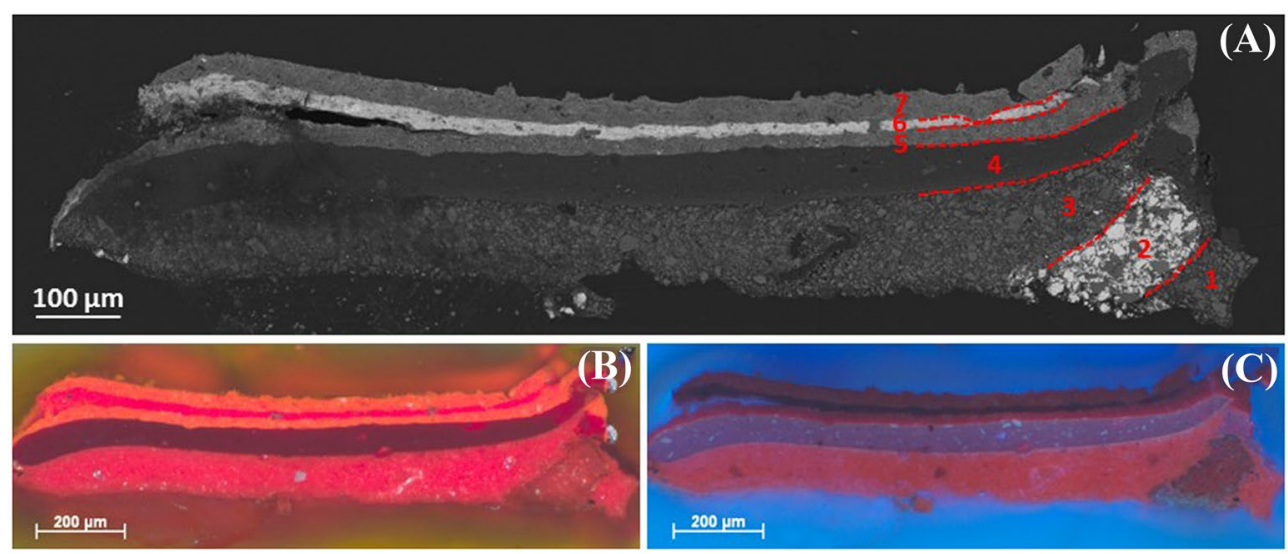

Fig. 7 BSE and optical microscopy images of cross section S11 (likely original and overpainting reds). a BSE image of cross section S11, i.e. multi-layered red paint removed from the sphere's red flange, revealing seven layers of likely original paint and overpaint. b Polarized and (c) UV light photographs of the same cross section, displaying different orange and red shades as well as fluorescence emission colors and intensities for the various paint layers

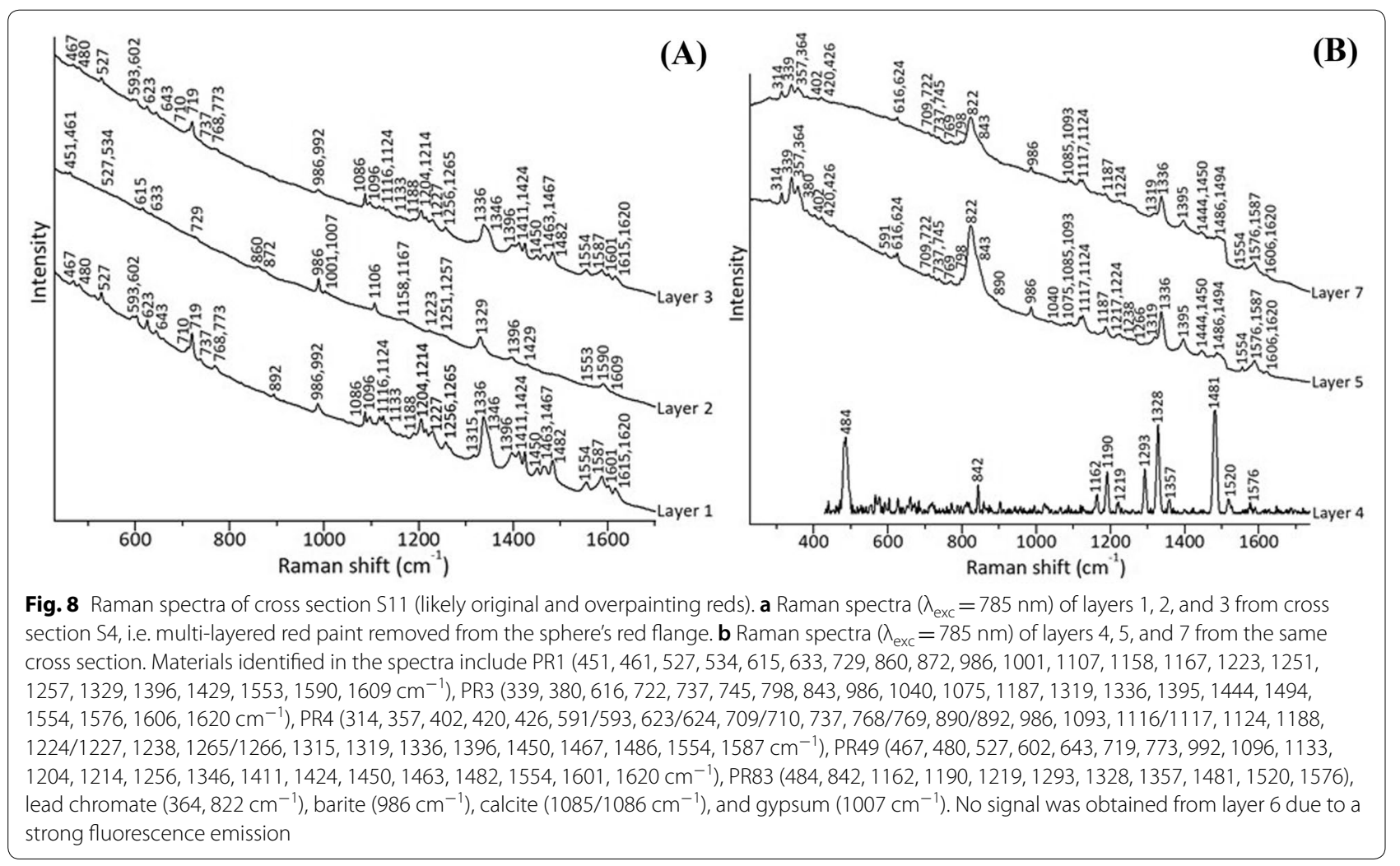

commonly employed in the production of low-cost printing inks for its brightness and bleed resistance, Lithol Red is extremely fugitive and its poor lightfastness has been identified as the cause of the dramatic color changes observed in artworks from the 1950s and 1960s, most notably paintings by Clyfford Still in The Met's collection
[23] and a mural cycle by Mark Rothko, known today as his Harvard Murals [22]. The other three $\beta$-naphthol reds identified in Calder's cross sections are also generally prone to fading when exposed to light, and some of them were originally developed for applications that did not only include artists' pigments. For instance, PR1, or Para 

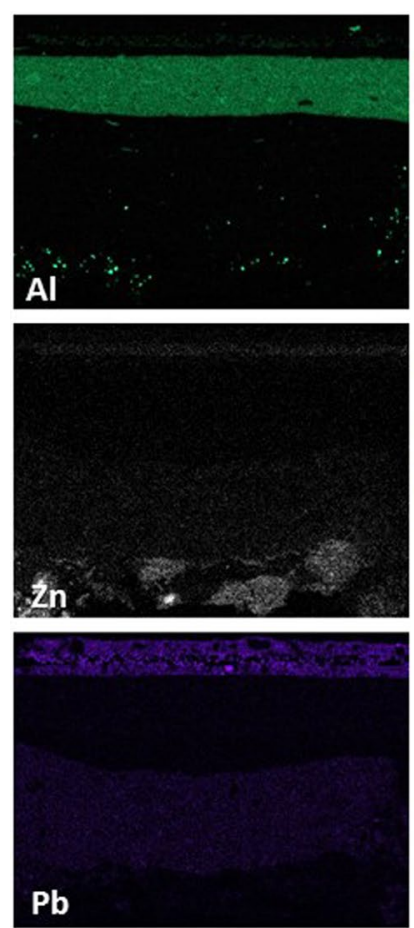
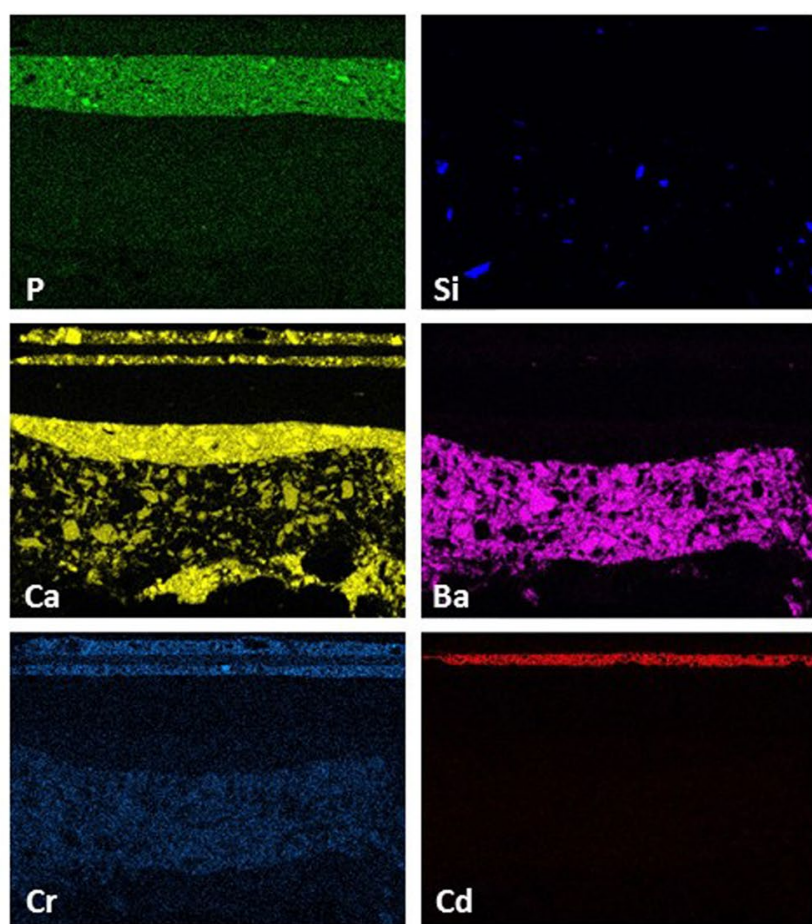
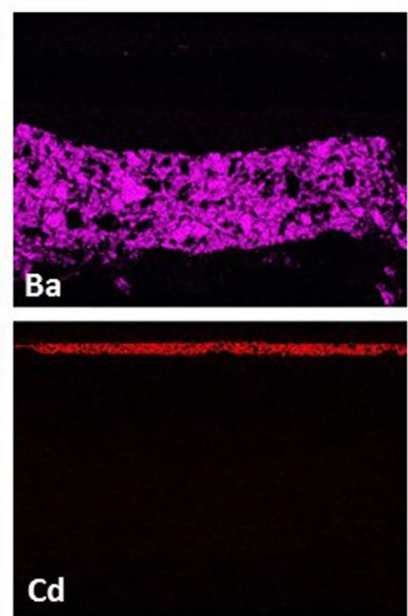
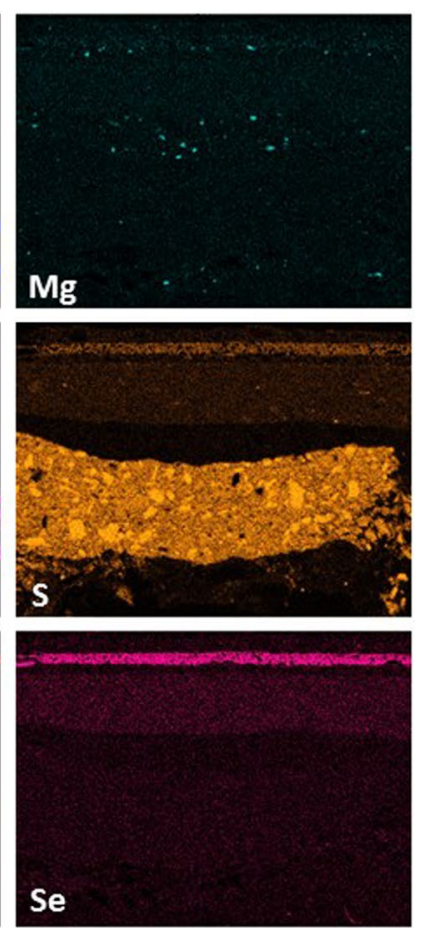

$100 \mu \mathrm{m}$

Fig. 9 X-ray elemental maps of selected major elements in cross section S12 (likely original and overpainting reds). X-ray elemental maps of selected major elements in a portion of cross section S12, i.e. multi-layered red paint removed from the sphere's red circle, showing the distribution of aluminum (Al), phosphorus (P), silicon (Si), magnesium (Mg), zinc ( $\mathrm{Zn})$, calcium (Ca), barium (Ba), sulfur (S), lead (Pb), chromium (Cr), cadmium $(\mathrm{Cd})$, and selenium (Se)

Red, first synthesized in 1880 by Holliday in England, is reported to have poor lightfastness and is not used in artists' paints because of bleeding; it was initially employed industrially in metal finishes and printing inks, but has been since replaced with more durable colors. PR3, also known as Toluidine Red, was first manufactured in 1904 by Lucius and Brüning in Germany, but reached its peak of popular use in the 1970s; also characterized by reduced resistance to light and weather, it is primarily employed for industrial coatings, in addition to wax crayons, pastels, and watercolors. PR4, or chlorinated Para Red, was first produced in the early 1900s along with its isomer PR6 (Parachlor Red). Despite its poor lightfastness and sensitivity to solvents, PR4 has been used extensively in artists' materials, including paints, colored pencils, and wax crayons [24, 25]. While the presence of additives and binders, among other factors, may affect the physicochemical properties of a paint, the overall unsatisfactory lightfastness of several of these pigments may partly justify the recurrent repainting observed on red areas of Calder's Half-Circle.

Binding medium analysis for samples S11 and S12 (all red paint layers combined) indicates another possible example of an early enamel, consisting of a mixture of oil, a diterpenoid resin from the Pinaceae family, and the polyhydric alcohol pentaerythritol (Fig. 10), a distinctive cross-linking agent used in place of or sometimes in mixture with glycerol from the mid-1940s [17]. While the fatty acid ratios $(\mathrm{P} / \mathrm{S}=0.85$ and $0.99, \mathrm{~A} / \mathrm{P}=1.9$ for both) suggest a drying oil, the presence of both marker compounds arachidic and behenic acids points to the addition of sunflower or safflower oil [26, 27], used in paints since 1949 [17]. However, in the case of modern oils, the addition of specific fatty acids to modify the oil's properties cannot be ruled out. Due to the inherent limitations of bulk analysis with Py-GC/MS in case of samples displaying a complex stratigraphy, it is difficult to draw conclusions on the exact dates of application of the numerous paint layers observed in these two cross sections. Indeed, while some of the pigments identified in S11 and S12 reached their peak of distribution in mid-20th century or later, they were all available in the early 1930s when Half-Circle was created. ATR-FTIR analysis of the stratigraphy of these samples did not detect binding media other than oil and resin in the bottom layer (layer 1); yet, based on the data collected, it is not possible to trace the 


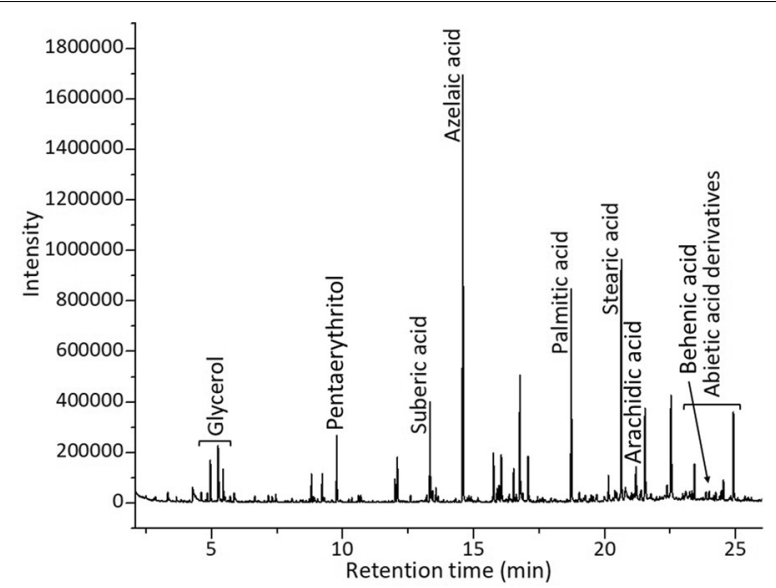

Fig. 10 Py-GC/MS chromatogram of sample S11 (likely original and overpainting reds). Py-GC/MS chromatogram obtained upon derivatization with TMAH of sample S11, i.e. multi-layered red paint removed from the sphere's red flange. Compounds identified in the chromatogram suggest the presence of early enamels, consisting of a mixture of modified drying oils and a diterpenoid resin from the Pinaceae family (palmitic acid methyl ester $74,87,43,55,41,143,75$, $57,69,227,270$; stearic acid methyl ester $74,87,43,55,75,143,41$, $57,69,298$; azelaic acid dimethyl ester $152,55,74,83,111,59,143$, 185, 69, 41, 217; methyl 6-dehydrodehydroabietate 237, 197, 41, 43, $195,155,141,165,179,312$; tetradehydroabietic acid, 7-methoxy-, methyl ester 342, 267, 282, 227, 343, 268, 228, 216, 251, 126;

15-methoxydehydroabietic acid, methyl ester 329, 330, 269, 313, 73, $344,43,107,145,314,344 ; 7,15$-dimethoxytetradehydroabietic acid, methyl ester 372, 297, 340, 313, 73, 357, 141, 341, 373, 257)

precise distribution of pentaerythritol and sunflower/safflower oil-the only components that may provide clues as to dating-within the layer structure. If one assumes the latter to be located in the upper portion of the cross sections, it is conceivable to expect for the first layers as being original, since enamel commercial paints were previously documented as Calder's paints [5-7]. On the other hand, it is likely that the paint layer(s) containing pentaerythritol and sunflower/safflower oil were applied as part of a later intervention.

\section{Black paint}

The black paint on Half-Circle's rod element is matte and appears to have been applied as a thin single layer directly on the metal; however, the lack of typical brush marks and its nearly pristine condition suggested that, while visually comparable to an original paint from the 1930s, it is likely a later repaint performed in an educated manner. With virtually no losses, wear, flaking or any signs of even partially failing adhesion of the paint to the steel support, such black paint likely constitutes a complete repaint carried out in the distant rather than recent past.

Black paint sample S13, visually thought to be a repainting layer, contains a carbon-based pigment and/ or bone/ivory black with calcite and gypsum dispersed in an early alkyd binder, based on the fatty acid ratios $(\mathrm{P} / \mathrm{S}=0.77, \mathrm{~A} / \mathrm{P}=1.1)$ as well as the presence of phthalic anhydride, bis (2-ethylhexyl) phthalate, pentaerythritol, and Pinaceae resin. While the fatty acid ratios are consistent with a drying oil, in this case, too, the presence of arachidic and behenic acid suggests that sunflower or safflower oil may have been added. Again, it should be noted that, in the case of modern oils, the addition of specific fatty acids to modify the oil's properties cannot be ruled out. Moreover, the detection of low amounts of elaidic, aleuritic, and butolic acids appear to indicate the use of shellac [28]. While the date of introduction of alkyd paints in America $[16,19,20]$ suggests that this paint layer may have been applied in or after the late 1930s, the presence of both glycerol and pentaerythritol as polyhydric alcohols pushes the earliest possible application date back to the second half of the 1940s [17].

\section{Conclusions}

The Whitney Museum of American Art, New York, owns one of the largest motorized works made by renowned American artist Alexander Calder, titled Half-Circle, Quarter-Circle, and Sphere and dated to 1932. This mobile sculpture, featured in the exhibition Calder: Hypermobility (June 9th-October 23rd, 2017), displayed several condition issues, including cease of motion of the motorized mobile and, most notably, the application of multiple repainting layers, resulting in a generally compromised appearance compared to the object's aesthetic as originally intended by the artist. Scientific analysis by means of a wide array of spectroscopic and chromatographic techniques was carried out with the main goal to gain insight into the stratigraphy of Half-Circle's painted surfaces, in terms of number and composition of the paint layers in selected white, red, and black colored areas, ultimately shedding light on the possible presence of an original paint layer. Results revealed, in most cases, the application of multiple layers of overpaint-in certain instances up to eleven - that contained a variety of modern materials, including titanium white in the form of tetragonal rutile, various synthetic organic red pigments, alkyd or late formulations of enamels based on orthophthalic acid/phthalic anhydride, glycerol and pentaerythritol, PVAc with plasticizers, and acrylics. However, some of the samples examined were found to be composed of materials that were available in the early 1930s, such as zinc white, calcite, and gypsum, as well as traditional drying oil binders, which supports the hypothesis that a layer of original paint may be present in certain areas of the work.

From a conservation standpoint, results of instrumental analysis reinforced the initial notions regarding 
Calder's paints in Half-Circle that were formulated based on visual observation. Most importantly, scientific data confirmed the presence of remnants of original paints below the current white and red colors, thus supporting the conservators' impetus for recovering layers believed to have been applied by the artist's hand. Further testing may be carried out in the near future to assess the extent and distribution of such underlying paints that are likely original, so that a proper treatment plan tailored to the removal of the overpaint may be formulated and optimized. Analysis of two other whites on the internal shelf and woodblock corroborated the initial visual assessment that these paints may belong to period recycled parts that Calder himself originally incorporated in the artwork's mechanism; therefore, such white layers will not be subjected to treatment. On the other hand, decisions regarding the existing non-original complete repaint of the black rod will be made after treatment of the white and red surfaces, upon visual re-evaluation of the black component in relation to the overall impact of the newly uncovered red and white.

To the authors' knowledge, the present work is the first technical investigation of one of Calder's indoor motorized sculptures ever reported in the literature. The data presented here may serve as a foundation for future studies on this groundbreaking artist's materials and techniques, as well as illuminate the historical information held in the various campaigns of overpainting that are frequently associated with Calder's works across museums' and private collections worldwide.

\section{Abbreviations \\ XRF: X-ray fluorescence spectroscopy; SEM/EDS: Scanning electron micros- copy with energy-dispersive X-ray spectroscopy; FTIR: Fourier-transform infrared spectroscopy; ATR-FTIR: Attenuated total reflection-Fourier-transform infrared spectroscopy; Py-GC/MS: Pyrolysis-gas chromatography/mass spectrometry.}

\section{Acknowledgements \\ The authors would like to thank Susan Braeuer Dam, Director of Research and Publications, Calder Foundation, and Steven Berger, electrician, who provided valuable resources and information for research and treatment. FP would also like to acknowledge Dr. Anna Cesaratto for her contribution to this project during her time at The Met working in the NICS program.}

\section{Authors' contributions}

FP carried out all scientific analysis, data interpretation, and drafted the manuscript. JA assisted with Py-GC/MS data interpretation and description of the corresponding results. EN provided art historical context, supported the scientific work, planned the conservation treatment, and helped with drafting the article's Introduction. All authors read and approved the final manuscript.

\section{Funding}

This research was made possible by the Network Initiative for Conservation Science (NICS), a Metropolitan Museum of Art program. Support for NICS was provided by a Grant (31500630) from The Andrew W. Mellon Foundation.

\section{Availability of data and materials}

All data generated during this study are included in this published article.

\section{Competing interests}

The authors declare that they have no competing interests.

\section{Author details}

${ }^{1}$ Department of Scientific Research, The Metropolitan Museum of Art, 1000 Fifth Avenue, New York, NY 10028, USA. ${ }^{2}$ Department of Conservation, Whitney Museum of American Art, 99 Gansevoort Street, New York, NY 10014, USA.

Received: 30 April 2020 Accepted: 20 July 2020

Published online: 08 August 2020

References

1. Calder CA. An autobiography with pictures with a new introduction by Jean Davison, 2 edn 1977. New York: Pantheon Books; 1966.

2. Calder A. Objects to art being static, so he keeps it in motion. New York: New York World-Telegram; 1932.

3. Osborn R. A conversation with Alexander Calder. Art in America Vol. 57, July-August, 1969; p. 31.

4. Calder A. Letter to "The Kieslers", March 12, 1932. Paris, Calder Foundation, Vignon file.

5. Bush K. Letter to Nancy McGary, then registrar at the Whitney Museum of American Art, June 15, 1987. New York, Whitney Museum of American Art, conservation files.

6. Lodge RG, Lodge EW. Notes for a history of "Calder Red" color and its paints in the United States in relation to the recoatings of Alexander Calder's Flamingo (1973) and La Grande Vitesse (1969) and other Calder stabiles; 1989.

7. Entry card for Alexander Calder. "Paint: T.J. Ronan, New York. Straight from the can, usually close to assumed standard for the primary colors. Source-artist; from OHR's (Orrin H. Riley) letter to Mr. Weil, Woodbourn Development Corp. St. Louis, Mo. of 1968; (date of card) entry: 5/16/69". New York, Solomon R. Guggenheim Museum, conservation files.

8. Bonaduce I, Burnstock A, Ormsby B, Scharff M, Carlyle L, Heydenreich G, Keune K. Conservation of modern oil paintings. van den Berg KJ, editor. Cham: Springer International Publishing; 2019.

9. Cappitelli F, Learner T, Chiantore O. An initial assessment of thermally assisted hydrolysis and methylation-gas chromatography/mass spectrometry for the identification of oils from dried paint films. J Anal Appl Pyrolysis. 2002;63:339-48.

10. Gardner HA. Physical and chemical examination of paints, varnishes, lacquers, and colors. 4th ed. Washington DC: Institute of Paint and Varnish Research; 1927. p. 664.

11. Tumosa CS. A brief history of aluminum stearate as a component of paint. Western Assoc Art Conserv Newslett. 2001;23(3):10-1.

12. Kokkori M, Sutherland K, Boon J, Casadio F, Vermeulen M. Synergistic use of Py-THM-GCMS, DTMS, and ESI-MS for the characterization of the organic fraction of modern enamel paints. Her Sci. 2015;3:30.

13. Sutherland K, Price B, Lins A, Passeri I. Oxalate-rich surface layers on paintings: implications for interpretation and cleaning. In: Mecklenburg MF, Charola AE, Koestler RJ, editors. New insights into the cleaning of paintings: proceedings from the cleaning 2010 international conference, Universidad Politécnica de Valencia and Museum Conservation Institute. Washington DC: Smithsonian Institution Scholarly Press; 2013. p. 85-7.

14. Rogge CE, Arslanoglu J. Luminescence of coprecipitated titanium white pigments: implications for dating modern art. Sci Adv. 2019;5(5):eaav0679.

15. Laver M. Titanium dioxide whites. In: West Fitzhugh E, editor. Artists' pigments. A handbook of their history and characteristics, vol. 3. Washington DC and Oxford: National Gallery of Art and Oxford University Press; 1997.

16. Learner TJS. Analysis of modern paints. Los Angeles: Getty Publications; 2004.

17. Standeven HAL. House paints, 1900-1960: history and use. Los Angeles: Getty Publications; 2011.

18. Kokkori M, Casadio F, Sutherland K, Vermeulen M. Charting the development of oil-based enamel paints through the correlation of historical paint technology manuals with scientific analysis. In: Burnstock A, de Keijzer M, Krueger J, Learner T, de Tagle A, Heydenreich G, van den Berg $\mathrm{KJ}$, editors. Issues in contemporary oil paint. Cham: Springer International Publishing; 2014. p. 117-25. 
19. Learner TJS, Smithen P, Krueger JW, Schilling MR, editors. Modern paints uncovered: proceedings from the modern paints uncovered symposium. Los Angeles: Getty Publications; 2007.

20. Crook J, Learner T. The impact of modern paints. New York: WatsonGuptill Publications; 2000.

21. Standeven HAL. The History and manufacture of Lithol Red, a pigment used by Mark Rothko in his Seagram and Harvard Murals of the 1950s and 1960s. Tate Papers. 2008;10:1-8.

22. Stenger J, Kwan EE, Eremin K, Speakman S, Kirby D, Stewart H, Huang SG, Kennedy AR, Newman R, Khandekar N. Lithol red salts: characterization and deterioration. e-PS. 2010;7:147-57.

23. Pozzi F. Report of Raman analysis for Clyfford Still paintings 1986.441 .6 and 1986.441.7. On file at the Department of Scientific Research, The Metropolitan Museum of Art; January 2016.

24. Quillen Lomax S, Learner T. A review of the classes, structures, and methods of analysis of synthetic organic pigments. J Am Inst Conserv. 2006;45:107-25.

25. Para Red and Toluidine Red. In: CAMEO: Conservation \& Art Materials Encyclopedia Online. Museum of Fine Arts, Boston. http://cameo.mfa. org/wiki/Main_Page. Accessed 3 Feb 2020
26. Izzo FC, van den Berg KJ, van Keulen H, Ferriani B, Zendri E. Modern oil paints -formulations, organic additives and degradation: some case studies. In: Burnstock A, de Keijzer M, Krueger J, Learner T, de Tagle A, Heydenreich G, van den Berg KJ, editors. Issues in contemporary oil paint. Cham: Springer International Publishing; 2014. p. 75-104.

27. Fuster-López L, Izzo FC, Piovesan M, Yusá-Marco DJ, Sperni L, Zendri E. Study of the chemical composition and the mechanical behaviour of $20^{\text {th }}$ century commercial artists' oil paints containing manganese-based pigments. Microchem J. 2016;124:962-73.

28. Sutherland K, del Río JC. Characterisation and discrimination of various types of lac resin using gas chromatography mass spectrometry techniques with quaternary ammonium reagents. J Chromat A. 2014;1338:149-63.

\section{Publisher's Note}

Springer Nature remains neutral with regard to jurisdictional claims in published maps and institutional affiliations.

\section{Submit your manuscript to a SpringerOpen ${ }^{\circ}$ journal and benefit from:}

- Convenient online submission

- Rigorous peer review

- Open access: articles freely available online

- High visibility within the field

- Retaining the copyright to your article

Submit your next manuscript at $\boldsymbol{\nabla}$ springeropen.com 